\title{
POLAR MOTION FROM LASER RANGE MEASUREMENTS OF GEOS-3
}

B. E. Schutz, B. D. Tapley, J. Ries

Department of Aerospace Engineering and Engineering Mechanics The University of Texas at Austin Austin, Texas

\section{ABSTRACT}

Using two-day arcs of GEOS-3 laser data, simultaneous solutions for pole position components, $x_{p}$ and $y_{p}$, and orbit elements have been obtained for the period spanning 3 February to 6 March 1976 using three NASA Goddard Space Flight Center laser stations located near Washington, D.C. (STALAS) and on the islands of Bermuda and Grand Turk. The results are in general agreement with the BIH results. However, because of the locations of the laser sites, the $x_{p}$ solution is weaker than the $y_{p}$ solution. The $x_{p}$ and $y_{p}$ estimates were smoothed with a straight line by weighted least squares using the variance associated with the pole estimates as weights in order to reflect the effect of widely different data distributions. The smoothed $y_{p}$ differs by one meter with respect to the BIH smoothed values and the smoothed $x_{p}$ differs by about two meters. Spectral analysis of the results has Identified frequencies associated with the orbital motion indicating the need for further improvements in the model of the physical system.

\section{INTRODUCTION}

The launch of the Geodynamics Experimental Ocean Satellite (GEOS-3) on April 9, 1975, initiated a priority laser tracking campaign to provide the necessary data for determining an accurate orbit of the satellite. These lasers included the NASA Goddard Space Flight Center (GSFC) systems which operated at a precision of 5 to $10 \mathrm{~cm}$, the Smithsonian Astrophysical Observatory (SAO) network of stations, and participating systems in other countries. Although the motivation for the laser deployment was to provide an accurate orbit for calibration and use of the GEOS-3 altimeter, it was apparent that contributions could be made to other applications requiring high precision such as polar motion. Previous polar motion experiments with laser systems were performed by Smith, et al. (1972) and Dunn, et al. (1977) using the Beacon ExplorerC Satellite to obtain one component of polar motion. Thus GEOS- 3 offered an opportunity to obtain polar motion results using intensive 
tracking from several high precision laser ranging systems.

A single laser range measurement may be described mathematically as a function of the geocentric coordinates of the satellite center of mass, the coordinates of the electronic center of the laser system, and a term representing contributions from the atmospheric refraction, noise and bias in the laser system, the correction between the reflector location and the center of mass of the satellite, errors resulting from an incomplete model of the dynamical system and the station coordinates errors. The commonly chosen origin for the reference geocentric coordinate system is the Conventional International Origin (CIO) as defined by the coordinates of the International Latitude Service stations. If the CIO system is used in conjunction with laser range measurements, an inherent problem exists to determine the laser coordinates in the CIO system.

Polar motion influences range measurements in two ways, viz., dynamically and kinematically. The former polar motion effect produces a force on the satellite as the result of changes in the inertial orientation of the geopotential. The latter effect is dominant and is dependent on the angle between the angular velocity vector of the Earth and the geocentric position vector of the observing station. As shown by Lambeck (1971), the kinematic influence on the laser range measurement includes a nearly diurnal signature. Consequently, a polar motion determination from artificial satellites requires proper modeling of all diurnal or nearly diurnal perturbations as well as other parameters which may appear to be diurnal as the result of non-uniform sampling of the orbit by the laser.

\section{GEOS- 3 MODEL AND DATA}

The GEOS-3 laser range data have been analyzed using the University of Texas Orbit Processor (Wilson, 1978) using a force model which included the GEM-10 geopotential (Lerch, et al., 1977), the luni-solar perturbations using DE-96 (Standish, et al., 1976), solid Earth tides with $\mathrm{k}_{2}=0.306$ (Farrell, 1972), atmospheric drag using Modified HarrisPriester (Dowd, 1977), and solar radiation pressure. The coefficient of drag and its time derivative were estimated in one set of solutions while $C_{D}$ was held fixed to 2.3 in a comparative set.

The laser measurement model used in the analysis included laser station coordinates from GEM-10, station tides with $h_{2}=0.615$ and $\ell_{2}=$ 0.084 (Gutenberg-Bullen A Earth mode1, Farrel1, 1972), tropospheric refraction corrections, laser reflector center-of-mass corrections, and appropriate timing corrections. Although a separate solution was obtained for station coordinates using various geopotentials, the GEM-10 set was adopted to maintain consistency with the geopotential, particularly with respect to terms producing signatures which are similar to polar motion. 
The laser data used in the analysis were obtained from three NASA Goddard Space Flight Center stations located at GSFC (STALAS) and on the islands of Bermuda and Grand Turk. The data analyzed were obtained during an intensive GEOS-3 tracking campaign from 3 February 1976 (760203) to 6 March 1976 (760306). This 32-day time period was divided into two-day arcs in which solutions for the pole components, $x_{p}$ and $y_{p}$ (assumed constant over the arc), and the orbital elements at the beginning of the arc were estimated. Two-day arcs were selected to ensure an adequate distribution of laser data in time while simultaneously reducing the effect of unmodeled orbital perturbations. Each arc was chosen to begin at $0^{\text {homos }} \mathrm{UTC}(\mathrm{BIH})$ on the appropriate date. For the sixteen arcs considered, laser measurements from all three stations were obtained within each arc except for the arcs beginning 760203, 760205, 760207,760302 and 760304 , in which no measurements from STALAS were obtained. On eleven occasions during this 32-day period, all three stations observed the same orbital revolutions. In addition, the measurement precision was less than ten centimeters and the systems pulsed once per second; however, an average pass produced between 150 and 200 measurements from a single station. Finally, although some GEOS-3 laser data were included in the GEM-10 geopotential and station coordinate solution, the February 1976 data were not used.

\section{RESULTS}

The estimates for the polar motion component $y_{p}$ are shown in Figure 1 , and Figure 2 gives the root mean square (rms) of the laser range residuals for each two-day arc. These solutions were obtained without constraints, i.e., the a priori error covariance matrix was assumed to be infinite. The BIH five-day smoothed values with straight line segments constructed over each five-day interval are also shown in Figure 1 for reference. To assist in the interpretation of the laser results, a straight line ( $a_{o}+a_{1} t$, where $a_{o}$ and $a_{1}$ are constants and $t$ is time) was fit to the pole estimates using a weighted least-squares method. The weighting parameters were the variances associated with the estimates for $x_{p}$ and $y_{p}$. These variances, which ranged from $(1 \mathrm{~cm})^{2}$ to (25 $\mathrm{cm})^{2}$ for $y_{p}$, do not account for errors introduced into the estimates when modeling errors exist, but are necessary to represent uncertainties in the estimates due to differing geographical and temporal distribution of data. The variance weighted rms yielded $1: 86 \mathrm{~m}$ for $\mathrm{x}_{\mathrm{p}}$ and $1.68 \mathrm{~m}$ for $\mathrm{y}_{\mathrm{p}}$. When $\overline{\mathrm{C}}_{\mathrm{D}}$ and $\dot{\mathrm{C}}_{\mathrm{D}}$ are estimated $\left(\mathrm{C}_{\mathrm{D}}=\overline{\mathrm{C}}_{\mathrm{D}}+\dot{\overline{\mathrm{C}}}_{\mathrm{D}} \mathrm{t}\right)$ the rms about the straight line fit was $1.86 \mathrm{~m}$ for $x_{p}$ and $1.36 \mathrm{~m}$ for $y_{p}$. The straight line smoothing of the $x_{p}$ estimates behave similarly to Figure 1 ; however, the fact that $x_{p}$ is approximately perpendicular to the meridians of the laser stations suggests that the estimates will be sensitive to small changes. This is further reflected in the variances associated with the estimates which show that the $x_{p}$ variance is usually greater than the $y_{p}$ variance. Figure 2 shows the overall effect of drag by including the rms of an estimation in which drag is not modeled. Similarly, the drag exclusion has a significant effect on the $x_{p}$ and $y_{p}$ estimates. Further details on these results are given by schutz, et al. (1978). 


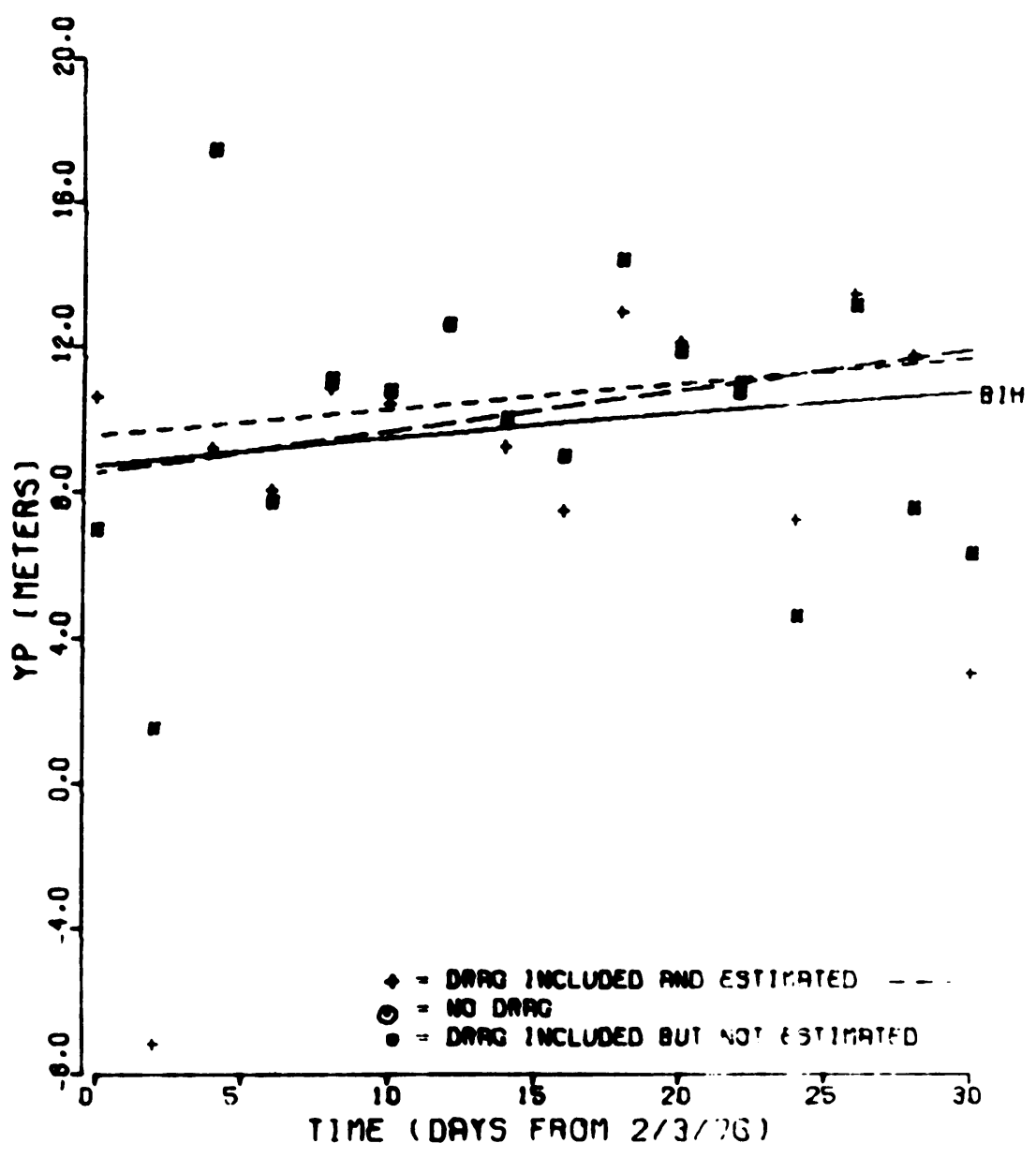

Figure 1. Estimates of $\mathrm{y}_{\mathrm{p}}$.

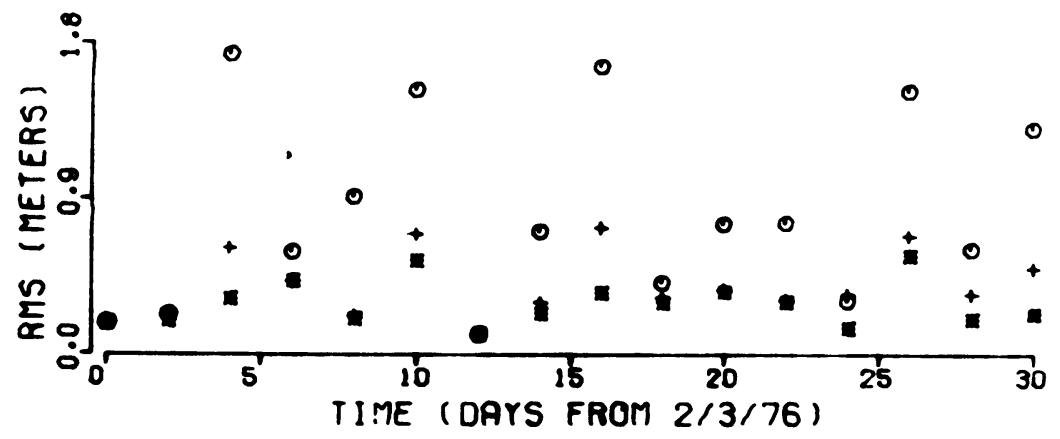

Figure 2. Rms of range residuals. 
It is significant to note that results have been obtained using GEOS-3 data between 27 August 1975 and 10 September 1975 (Schutz, et al., 1978) which have also demonstrated the ability to estimate mean values of pole position which are in agreement with the BIH. For comparison, the results during this 1975 period produced a weighted rms of about $85 \mathrm{~cm}$ in the y component. The February results presented in this paper are especially significant since these data were not used in the GEM-10 geopotential and station coordinate solution, thus demonstrating an ability to predict the pole position which further reflects the overall model consistency.

There are two possible sources for the apparent systematic trends exhibited in Figure 1. Since the astronomical data provide no evidence for supporting the general behavior in this figure, most of the behavior must be attributed to unmodeled orbit perturbations. These perturbations exhibit frequencies similar to polar motion over the time period of their estimation, thus aliasing the $x_{p}$ and $y_{p}$ estimates. Further analysis was performed through use of the maximum entropy spectral analysis of Burg (1977). This analysis identified significant frequencies of about the same power at periods of $4.8^{\mathrm{d}}, 5.5^{\mathrm{d}}, 10.7^{\mathrm{d}}$, and $16^{\mathrm{d}}$. In addition, a $7 \mathrm{~d}$ period was identified with three times the power of the other frequencies. Since the resonance period of GEOS- 3 due to fourteenth order terms in the geopotential is approximately $4.5 \mathrm{~d}$, the 4.8d period must be associated with "mismodeling" of these terms. Furthermore, the $10^{\mathrm{d}}$ and $16^{\mathrm{d}}$ periods can be correlated with ocean tide perturbations (Goad, 1977), leaving only the $5.5^{\mathrm{d}}$ and $7 \mathrm{~d}$ periods of unknown origin.

Although a very significant earthquake occurred in Guatemala on 4 February 1976 at $9^{\mathrm{h}}$ UT, there is no immediate correlation of the pole coordinates associated with the 760205 arc which significantly differ from the other values in February. Other earthquakes of similar magnitude occurred in this time period without comparable changes in pole position. Thus the observed behavior must be attributed to temporal and geographical data distribution.

\section{CONCLUSIONS}

These polar motion results have been obtained using regional tracking from three laser systems deployed off the U. S. East coast for GEOS-3 experiments. The results obtained have demonstrated the ability of the dynamical model and laser station coordinates to track the pole position with an uncertainty of less than two meters. This uncertainty indicates that additional model improvements are necessary for further analysis of GEOS-3 data. However, the results are significant in the implication that higher altitude satellites such as LAGEOS, which are substantially less dependent on model uncertainties from sources such as drag and geopotential, will provide polar motion estimates commensurate with the measurement precision. 


\section{ACKNOWLEDGEMENTS}

This investigation was supported by the National Aeronautics and Space Administration through contract NAS 6-2454. The contributions of $\mathrm{Mr}$. R. Eanes, T. Wilson, J. Lundberg, and C. Shum are gratefully acknowledged.

\section{REFERENCES}

Burg, J. P.: 1977, Paper presented at the 37th Ann. Int. Meeting Soc. of Explor. Geophys., Oklahoma City.

Dowd, D. L.: 1977, Institute for Advanced Study in Orbital Mechanics Tech. Rep. 77-4, The University of Texas at Austin.

Dunn, P. J., Smith, D. E.: 1977, J. Geophys. Res. 82, pp. 895-897.

Farre11, W. E.: 1972, Rev. of Geophysics and Space Physics 10, pp. 761797.

Goad, C.: 1977, U. S. Department of Commerce, NOAA Technical Report NOS 71 NGS 6.

Lambeck, K.: 1971, Bulletin Geodesique, pp. 263-281.

Lerch, F., Klosko, S., Laubscher, R., and Wagner, C.: 1977, Goddard Space Flight Center Report X-921-77-246.

Schutz, B. E., Tapley, B. D., and Ries, J.: 1978, Institute for Advanced Study in Orbital Mechanics Tech. Rep. 78-4.

Smith, D. E., Kolenkiewicz, R., Dunn, P. J., Plotkin, H. H., Johnson, T. S.: 1972, Science 178, pp. 405-406.

Standish, E. M., Keesey, M., and Newhal1, S.: 1976, Jet Propulsion Laboratory Tech. Rep. 32-1063.

Wilson, T.: 1978, Institute for Advanced Study in Orbital Mechanics Tech. Rep. 78-1.

\section{DISCUSSION}

H. G. Walter: Could you comment on your adjustment of the Love number which is significantly different from that of the Goddard Space Flight Center group?

B. E. Schutz: No Love number adjustment was performed. Instead, $k_{2}$, $\mathrm{h}_{2}$, and $1_{2}$ were adopted from Farrell's Gutenberg-Bullen A Earth model. Furthermore, the results presented will not change at the decimeter level or less as the result of small perturbations in $k_{2}$, $\mathrm{h}_{2}$, and $\mathrm{l}_{2}$. 